\title{
Development of a High Spatial Resolution SQUID Magnetometer for Biomagnetic Measurement
}

\author{
Koichiro Kobayashi and Yoshinori Uchikawa, Member, IEEE
}

\begin{abstract}
Some high resolution superconducting quantum interference device (SQUID) magnetometers have been developed designed for nerves, muscles, slices of brain tissue and small animals. In these SQUID system, diameters of the pickup coil were a few millimeters and the distances between the pickup coil and the outside surface of the dewer were a few millimeters. We developed a super high resolution SQUID magnetometer for biomagnetic measurement having higher spatial resolution than previous systems. In this system, diameter of the pickup coil is $200 \mu \mathrm{m}$, the distance between the pickup coil and the outside surface of the dewar is $800 \mu \mathrm{m}$. The typical system noise in the magnetic shielded room is $2.7 \mathrm{pT} / \sqrt{\mathrm{Hz}}$ in the frequency of the white-noise region.

The spatial resolution of this SQUID system is analyzed using are demonstrated on a current source model and MCG measurements of a human and a frog. The magnetic field pattern is able to separate the meander line of $500 \mu \mathrm{m}$ interval.
\end{abstract}

Index Terms-Biomagnetism, magnetocardiogram, SQUID magnetometer.

\section{INTRODUCTION}

$\mathbf{S}$ INCE 1970, superconducting quantum interference device (SQUID) magnetometers have been used for biomagnetic studies [1]. It is especially necessary for use in medical diagnosis and the study of higher brain functions. The main aim of biomagnetic measurement is to estimate the sources of electrical activity in the brain and the heart. On the other hand, for advancement of clinical applications of the magnetoencephalogram (MEG) and the magnetocardiogram (MCG), it is necessary to investigate the relationship between diseases and induced biomagnetic fields using an experimental animal model. Typical SQUID magnetometers are constructed with $15-25 \mathrm{~mm}$ diameter pickup coils. This type configuration of pickup coil is designed for the human brain and heart. Because of limited spatial resolution, this configuration cannot be used for the MEG and MCG measurements of small animals such as the rat the frog. A high resolution magnetometer is needed to measure the biomagnetic fields produced by small animals in conjunction with more invasive physiologic measurements. Wikswo [2], Buchanan [3], Okada [4] and Iramina [5] et al. developed high resolution SQUID magnetometers, composed of a few pickup coils that were 3-5 mm in diameter, which was designed for the experiments on in vitro preparations such as isolated nerves and

Manuscript received January 4,2003 . This work was supported in part by a Grant-in-Aid for the High-Technology Research Center, Tokyo Denki University.

K. Kobayashi is with the Faculty of Engineering, Iwate University, Iwate, Japan (e-mail: kobaya@iwate-u.ac.jp).

Y. Uchikawa is with the Faculty of Science and Engineering, Tokyo Denki University, Saitama, Japan (e-mail: uchikawa@f.dendai.ac.jp).

Digital Object Identifier 10.1109/TMAG.2003.816156

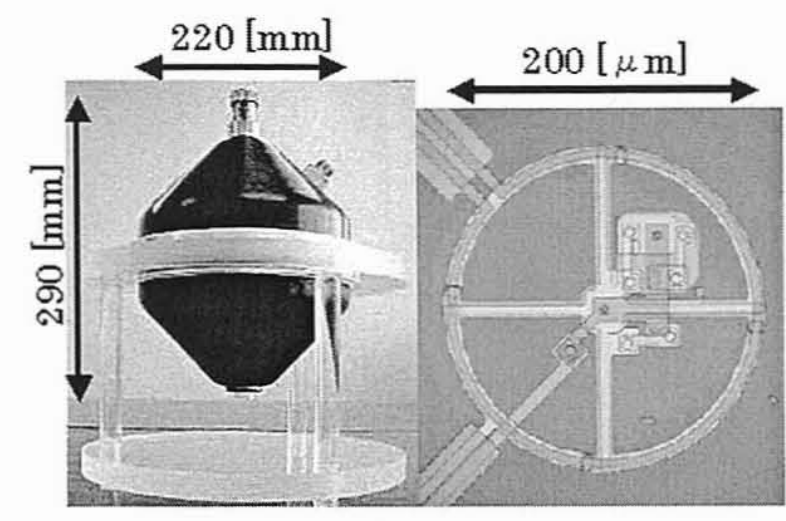

(a) the dewar

(b) the pickup coil

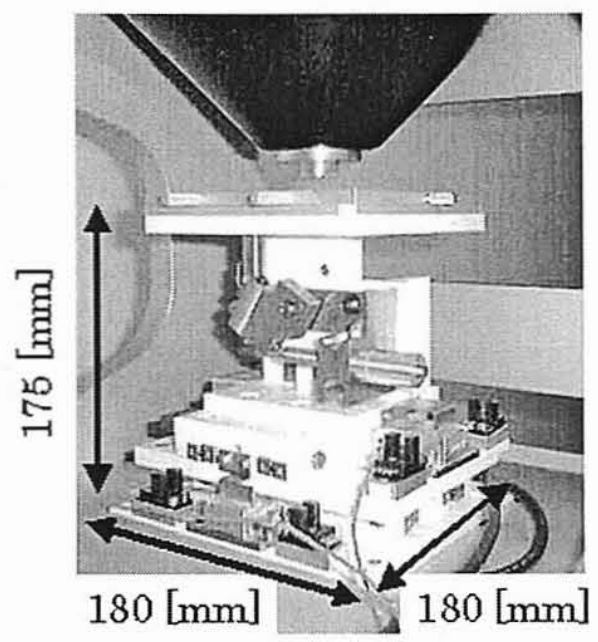

(c) the XYZ-stage

Fig. 1. Dewar, pickup coil and the XYZ-stage: (a) the dewar, (b) the pickup coil, and (c) the XYZ-stage.

muscles and slices of brain tissue and small animals. Most recently, Baudenbacher et al. developed a super high resolution SQUID magnetometer, composed of two type pickup coils that were $250 \mu \mathrm{m}$ and $500 \mu \mathrm{m}$ in diameter, which was designed for the imaging biomagnetic fields of room temperature samples with sub-millimeter resolution [6].

In this study, we developed a high resolution SQUID magnetometer for biomagnetic measurement having higher spatial resolution than previous systems. We discuss the spatial resolution of this SQUID system obtained from a current source model and demonstrate the MCG measurements of a human and a frog. 


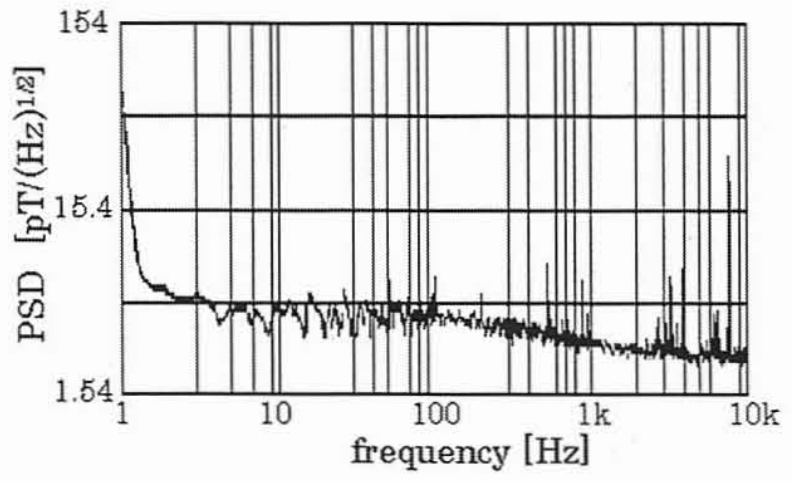

Fig. 2. Power spectrum of environmental magnetic noise in the magnetically shielded room.

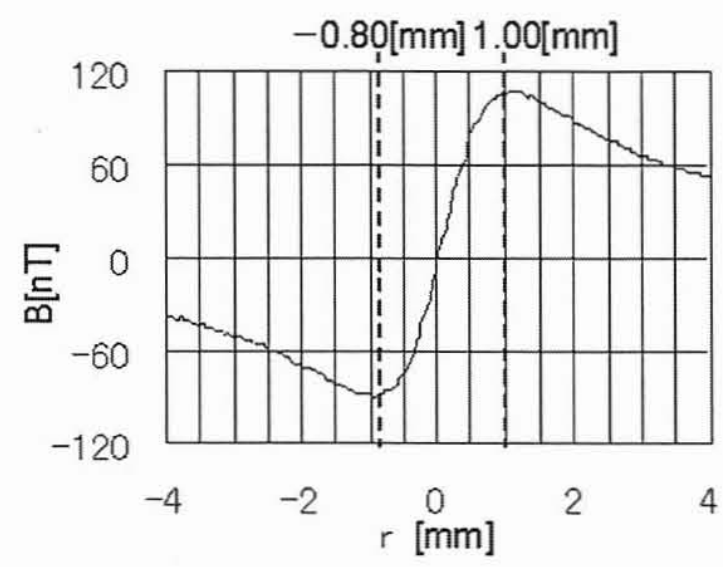

Fig. 3. Measured magnetic field from a current line. (measured magnetic field $\mathrm{B}$ versus measurement position $\mathrm{r}$ ).

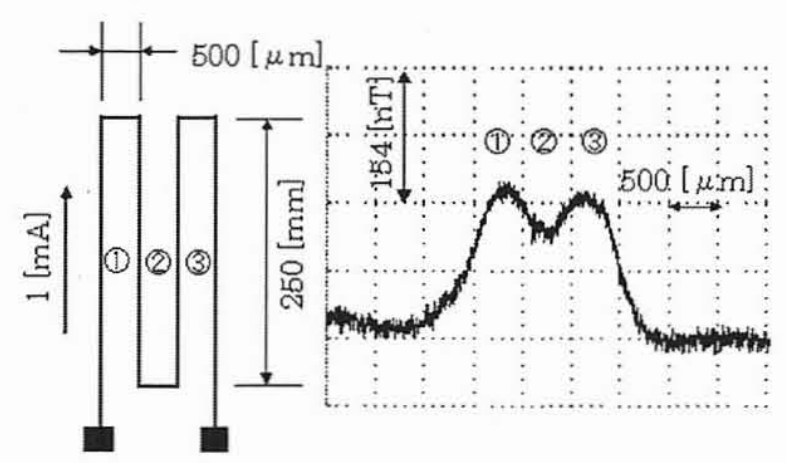

$\begin{array}{ll}\text { (a) the meander line of } 500 \mu \mathrm{m} \text { interval } & \text { (b) the magnetic field pattern }\end{array}$

Fig. 4. Magnetic field pattern obtained from the meander line of $500 \mu \mathrm{m}$ interval: (a) the meander line of $500 \mu \mathrm{m}$ interval and (b) the magnetic field pattern.

\section{Measurement System}

The SQUID system consists of single channel magnetometer of low temperature type. Fig. 1 shows the dewar, the pickup coil and the XYZ-stage. The diameter of the pickup coil is $200 \mu \mathrm{m}$. The dewar is $290 \mathrm{~mm}$ height, $220 \mathrm{~mm}$ width and made chiefly from the aluminum. The dewar has a capacity of 11 and allows

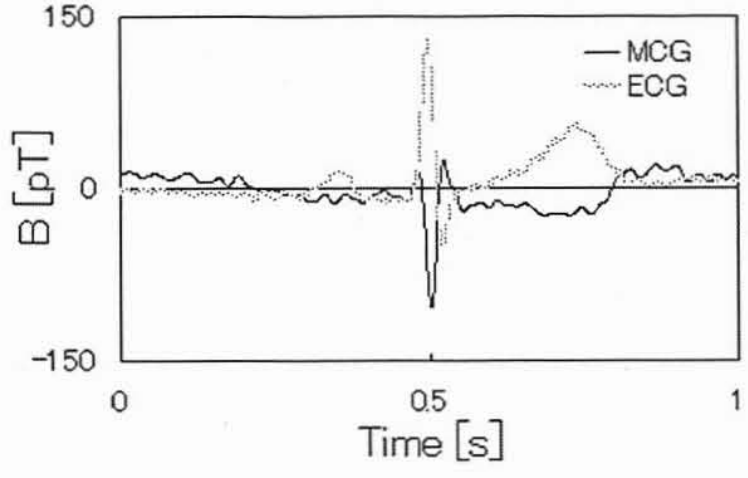

(a) The MCG and ECG waveforms of the human, measurement position is at right atrium

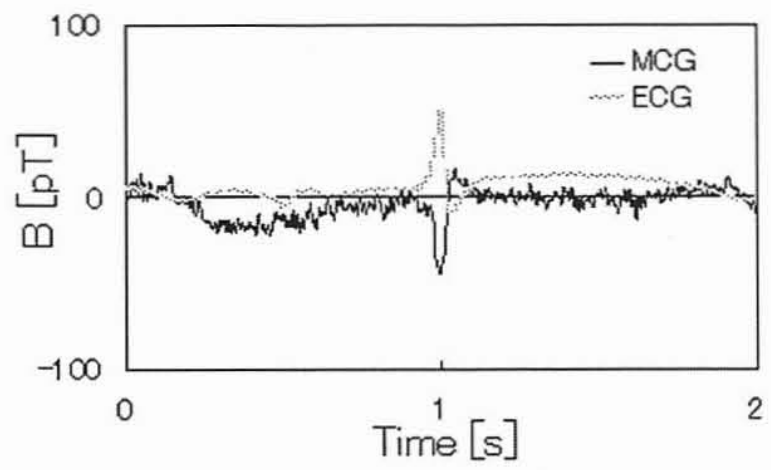

(b) The MCG andECG waveforms of the frog, measurement position is at right atrium

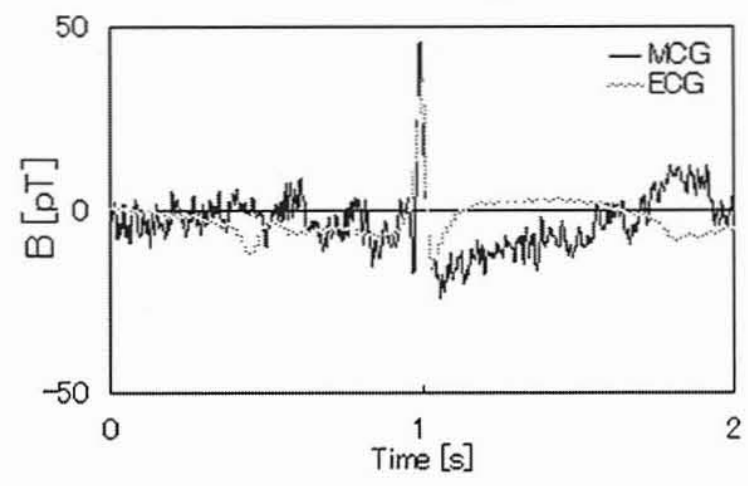

(c) The MCG and ECG waveforms of the frog, measurement position is at right ventricle

Fig. 5. Averaged MCG and ECG waveform of the human and the frog: (a) MCG and ECG waveforms of the human, measurement position is at right atrium, (b) MCG and ECG waveforms of the frog, measurement position is at right atrium, and (c) MCG and ECG waveforms of the frog, measurement position is at right ventricle.

for an operation of the SQUID magnetometer for about $10 \mathrm{~h}$ without replenishing the cryogen. The XYZ-stage made from the ceramic, brass and aluminum. The XY directions of the stage are driven by the nonmagnetic piezoelectric motors with high precision optical encoders. The $\mathrm{Z}$ direction of the stage is driven with a manually micrometer. The scanning range is $60 \mathrm{~mm}$ in $\mathrm{XY}$ direction with an absolute resolution of $1 \mu \mathrm{m}$. The intrinsic equivalent flux noise of the SQUID is about $9.1 \mu \Phi_{0} / \sqrt{ } \mathrm{Hz}$. 
Fig. 2 shows the power spectrum of environmental magnetic noise in the magnetically shielded room. The typical system noise in the magnetic shielded room is $2.7 \mathrm{pT} / \sqrt{\mathrm{Hz}}$ in the frequency of the white-noise region.

The magnetic field from a current line was measured to examine the distance between the pickup coil and the outside surface of the dewar. Fig. 3 shows the measured magnetic field from a current line. The current line is set on the stage left $0.1 \mathrm{~mm}$ from the dewer. $\mathrm{X}$-axis shows the measurement position, $\mathrm{Y}$-axis shows the measured magnetic field. The distance between pickup coil and the current line is a half of the distance between maximum value position and minimum value position of measured magnetic field. Therefore, in this SQUID magnetometer, the distance between the pickup coil and the outside surface of the dewar becomes $(1.0-(-0.8)) / 2-0.1=0.8 \mathrm{~mm}(800 \mu \mathrm{m})$.

In order to examine the spatial resolution of this SQUID magnetometer, the magnetic field from the meander line of $500 \mu \mathrm{m}$ interval shown in Fig. 4(a) was measured. Fig. 4(b) shows the magnetic field pattern obtained from the meander line of $500 \mu \mathrm{m}$ interval. We can clearly see two positive extremes (1), (3)) in Fig. 4(b). The spatial resolution is less than $500 \mu \mathrm{m}$.

\section{MCG MEASUREMENT}

MCGs of a human and a frog were measured by this system. A band-pass filter was used in the range of 0.1 to $100 \mathrm{~Hz}$. All magnetic data were averaged for 100 times at each position. The lead II of ECG was measured same time. The reference signal for averaging was R-wave of ECG.

Fig. 5(a) shows an example of the MCG and ECG waveforms recorded from the human, the measurement position is at right atrium. Fig. 5(b) and (c) show examples of the MCG and ECG waveforms recorded from the frog, measurement positions are at right atrium and right ventricle. In these waveforms, we are able to see the QRS complex. The MCG measurement such as small animal is difficult to see the inversion of the polarity of the QRS complex using typical SQUID magnetometers $(20 \mathrm{~mm}$ diameter pickup coil), because of limited spatial resolution. On the other hand in Fig. 5(b) and (c) the inversion of the polarity at the QRS complex of MCG is discernible.

\section{CONCLUSION}

We developed a super high resolution SQUID magnetometer for biomagnetic measurement having higher spatial resolution than previous systems. In this system, diameter of the pickup coil is $200 \mu \mathrm{m}$, the distance between the pickup coil and the outside surface of the dewar is $800 \mu \mathrm{m}$. We showed that the spatial resolution of the magnetic field obtained from the meander line is less than $500 \mu \mathrm{m}$. We demonstrated the MCG measurements of à human and a frog. It is possible to see the inversion of the polarity at the QRS complex of the frog's MCG waveforms.

\section{REFERENCES}

[1] D. Cohen, E. A. Edelsack, and J. E. Zimmerman, "Magnetocardiograms taken inside a shielded room with a superconducting pointcontact magnetometer," Appl. Phys. Lett., vol. 16, pp. 278-280, 1970.

[2] J. P. Wikswo, R. N. Friedman, A. W. Kilroy, J. M. V. Egeraat, and D. S. Buchanan, "Preliminary measurements with microSQUID," in $A d$ vances in Biomagnetism. New York: Plenum, 1989, pp. 681-684.

[3] D. S. Buchanan, D. B. Crum, D. Cox, and J. P. Wikswo, "MicroSQUID a close-spaced four channel magnetometer," in Advances in Biomagnetism. New York: Plenum, 1989, pp. 677-679.

[4] Y. C. Okada, S. Kyouhou, A. Lahteenmaki, and C. Xu, "A high-resolution system for magnetophysiology and its applications," in Biomagnetism: Clinical Aspects. Amsterdam, The Netherlands: Elsevier, 1992, pp. 375-383.

[5] K. Iramina, B. Hong, S. Uchida, K. Goto, and S. Ueno, "Measurements of biomagnetic fields using a high-resolution dc superconducting quantum interference device magnetometer," J. Appl. Phys. AIP, vol. 83 , no. 11 , pp. $6465-6467,1998$.

[6] F. Baudenbacher, N. T. Peters, P. Baudenbacher, and J. P. Wikswo, "High resolution imaging of biomagnetic fields generated by action currents in cardiac tissue using a LTS-SQUID microscope," Phys. C, vol. 368, pp. 24-31, 2002. 